\title{
Characterization of Endophytes Isolated from Eclipta prostrata and Roles in Regulating the Gut Microbiota of C57BL/6J Mice
}

\author{
Xin Zhao ${ }^{1,2}$, Huishu Shi ${ }^{3}$, Yajing Wang ${ }^{1,3 *}$ \\ ${ }^{1}$ Institute of Traditional Chinese Medicine, Tianjin University of Traditional Chinese Medicine, Tianjin, China \\ ${ }^{2}$ Key Laboratory of Pharmacology of Traditional Chinese Medical Formulae, Ministry of Education, Tianjin University of \\ Traditional Chinese Medicine, Tianjin, China \\ ${ }^{3}$ Engineering Research Center of Modern Chinese Medicine Discovery and Preparation Technique, Ministry of Education, Tianjin \\ University of Traditional Chinese Medicine, Tianjin, China \\ Email: *yajing022@163.com
}

How to cite this paper: Zhao, X., Shi, H.S. and Wang, Y.J. (2020) Characterization of Endophytes Isolated from Eclipta prostrata and Roles in Regulating the Gut Microbiota of C57BL/6J Mice. Advances in Bioscience and Biotechnology, 11, 166-187.

https://doi.org/10.4236/abb.2020.115014

Received: April 12, 2020

Accepted: May 11, 2020

Published: May 14, 2020

Copyright (c) 2020 by author(s) and Scientific Research Publishing Inc. This work is licensed under the Creative Commons Attribution International License (CC BY 4.0).

http://creativecommons.org/licenses/by/4.0/

\begin{abstract}
Eclipta prostrata has been extensively studied for its promoting effect on human health. Previous study proved that E. prostrata possessed anti-osteoporosis function in SAMP6 mice via gut microbiota (GM). Endophytes are widespread in plants, especially in Chinese herbal medicines. However, little is known regarding the endophytes of E. prostrata. In this study, we focus on screening and identifying the endophytes from plant $E$. prostrata and exploring their roles in modulating GM. According to biochemical, physiological tests and $16 \mathrm{~S}$ rDNA sequence analysis, ten endophytes were characterized in different locations of plant E. prostrata belonging to Lactococcus, Bacillus, Enterococcus, Exiguobacterium and Pantoea. The antimicrobial activity of endophytes EP01-10 was investigated via the oxford cup method. Furthermore, the acid and bile salt resistant ability of EP01-10 was detected to explore their survival ability in gastrointestinal tract. Results indicated that strains of Lactococcus, Bacillus and Exiguobacterium (EP01, 03 and 05) showed strong antimicrobial activity against Escherichia coli and Staphylococcus aureus and strains of Lactococcus, Bacillus and Enterococcus (EP01, $02,04,08$ and 10) have the ability to survive in the gastrointestinal tract. $16 \mathrm{~S}$ rRNA sequencing of GM in C57BL/6J mice was performed for diversity and structure analysis responded to the administration of $E$. prostrata extract. $E$. prostrata extract acted on improving the microflora diversity, particularly in increasing the abundance of Lactococcus significantly. Thus, as an endophyte, Lactococcus plays an important role in E. prostrata modulating intestinal tract. Our study suggested that endophytes of Chinese herbal medicines
\end{abstract}


might be a novel target for the treatment of diseases by regulating the GM.

\section{Keywords}

Endophyte, Eclipta prostrata, Gut Microbiota, C57BL/6J Mice, Lactococcus

\section{Introduction}

Increasing studies have confirmed that a number of Chinese herbs could regulate the balance of intestinal micro-ecology [1] [2]. Endophytes and their metabolites of Chinese herbs play an important role in the field of agriculture, food, medicine, ecology and have a wide range of antimicrobial activities [3] [4] [5]. The antimicrobial substances of endophytes have low cytotoxicity and are mostly novel [6]. In addition, endophytes can regulate the biosynthesis of plant secondary metabolites [7]. Endophytes including Bacillus, Enterobacter and Arthrobacter, are ubiquitous in plant roots and stems [8]. There were also endophytes from rhizosphere, which is close to common bacteria in soil [9]. Producing antibiotics or plant-promoting biomass plays a significant role in biological control, nitrogen fixation and plant growth [10]. However, the screening of endophytes and how they involve in plants to perform their biological functions in gut remain to be studied.

Eclipta prostrata, a traditional Chinese medicine (TCM) of sunflower family, has been used in China for treating uterine bleeding, loose teeth, tinnitus and dizziness [11] [12] [13]. The E. prostrata extract (by EtOH) used in this study was qualitatively and quantitatively analysed by UHPLC-Q-TOF-MS and UPLC-MS/MS, respectively [14]. The content of seven main compounds in $E$. prostrata extract was as follows: $0.233 \pm 0.005 \mathrm{mg} \cdot \mathrm{g}^{-1}$ (ecliptasaponin IV), 1.021 $\pm 0.004 \mathrm{mg} \cdot \mathrm{g}^{-1}$ (ecliptasaponin A), $0.272 \pm 0.003 \mathrm{mg} \cdot \mathrm{g}^{-1}$ (apigenin), $0.115 \pm 0.003$ $\mathrm{mg} \cdot \mathrm{g}^{-1}$ (3'-hydroxybiochanin A), $0.306 \pm 0.004 \mathrm{mg} \cdot \mathrm{g}^{-1}$ (luteolin), $0.125 \pm 0.008$ $\mathrm{mg} \cdot \mathrm{g}^{-1}$ (luteolin-7-O-glucoside), $0.271 \pm 0.001 \mathrm{mg} \cdot \mathrm{g}^{-1}$ (wedelolactone). Pharmacological studies have shown that E. prostrata extract displays anti-osteoporosis effects by modulating gut microbiota (GM) [15]. E. prostrata contains such various active ingredients widely distributing in plants and might contribute to a good environment for endophytes.

In this study, various endophytes were isolated from wild plant samples of $E$. prostrata, some of which had significant inhibitory effects on Staphytococcus aureus and Escherichia coli. However, the relationship of endophytes of E. prostrata and GM is unclear. Based on the survival ability in gastrointestinal tract, endophytes of $E$. prostrata were further analysed. $16 \mathrm{~S}$ rRNA sequencing was conducted to assess the effect of $E$. prostrata on the GM community and endophytes involved in regulating the GM of mice would be figured out. Screening and charactering endophytes from TCM is a hot field, which expand the study of pharmacodynamics and underlying mechanism via GM. 


\section{Methods and materials}

\subsection{Microorganisms and Culture Conditions}

The microorganism EP01-10 listed in Table 1 used in this study isolated from $E$. prostrata herbs sample including six Bacillus strains, one Lactococcus strain, one Exiguobacterium strain, one Pantoea strain, one Bacterium strain and the indicator strains of E. coli CMCC44103 and S. aureus ATCC6358 were preserved at microbiology laboratory of Tianjin University of traditional Chinese medicine. All the medium components used in this study were reagent pure grade purchased from Solarbio Co., Ltd. (Beijing, China). All other chemicals used were of analytical grade unless otherwise stated. Solid media used in the antibacterial study consisted of MRS agar (potato, $200 \mathrm{~g}$; glucose, $20 \mathrm{~g}$; agar, $18 \mathrm{~g}$; and distilled water, $1 \mathrm{~L}$ ) and Luria-Bertani agar (pep-tone, $10 \mathrm{~g}$; yeast extracts, $5 \mathrm{~g} ; \mathrm{NaCl}, 10 \mathrm{~g}$; agar, $18 \mathrm{~g}$; and distilled water, $1 \mathrm{~L}$ )., the same agar-lacking liquid media, were used for fermentation test. The strain EP01-10 was activated by transferring single colonies of the strain from plates to $10 \mathrm{~mL}$ activation LB or MRS medium. The flasks were shaken at $37^{\circ} \mathrm{C}, 150 \mathrm{rpm}$ for $16 \mathrm{~h}$.

\subsection{Experimental Animals}

Experimental animals including 2-month-old female healthy mice C57BL/6J (SPF level) were provided by Huafukang Co., Ltd. (Beijing, China). Animals were raised in institute of radiation medicine Chinese academy of medical sciences (Tianjin, China), at room temperature $\left(22^{\circ} \mathrm{C} \pm 2^{\circ} \mathrm{C}\right)$, with relative humidity of $58 \%-65 \%$ and light cycle of $12 \mathrm{~h}$ alternation. In this study, 10 same-treated mice were tested for each group ( 5 per cage).

\subsection{Preparation of Raw Material and dose of E. prostrata}

E. prostrata were collected in April 2018 from the Jiulongshan of Qingyuan

Table 1. Colony characteristics of endophytic strains in different location of E. prostrata.

\begin{tabular}{lccc}
\hline Name & Location in E. prostrata & Colony Characteristics & The highest homology strain \\
\hline EP01 & Root & medium size, raised, slightly white, moist, with neat edges & Lactococcus sp. ARa2 \\
EP02 & Leaf & white, round, smooth and opaque, with neat edges & Enterococcus faecium \\
EP03 & Stem & white, round, smooth and opaque, with neat edges & Bacillus megaterium \\
EP04 & Root & white, round, smooth and opaque, with neat edges & Bacillus sp. WS22 \\
EP05 & Leaf & yellow, smooth, edge regular, protruding & Exiguobacterium acetylicum \\
EP06 & Root & white, round, smooth and opaque, with neat edges & bacterium L35 \\
EP07 & Stem & round, yellow, edge neat, low convex, smooth & Pantoea agglomerans \\
EP08 & Leaf & white, round, smooth and opaque, with neat edges & Bacillus megaterium \\
EP09 & Root & white, round, smooth and opaque, with neat edges & Bacillus aryabhattai \\
EP10 & Root & colorless, transparent or translucent, relatively flat, & Bacillus cereus
\end{tabular}


(Guangdong, China). The surface of fresh E. prostrata was cleaned with sterile water and dried at room temperature. Under sterile conditions, $100 \mathrm{mg}$ of roots, stems and leaves were cut into tubes, respectively. $1 \mathrm{~mL}$ of sterile miliQ water was added, and samples were centrifuged at $4000 \mathrm{rpm}$ for $1 \mathrm{~min}$. The supernatant was collected for the following endophytes screening.

According to previous study [15], $2 \mathrm{~kg}$ of dried E. prostrata was refluxed by $70 \% \mathrm{EtOH}$ for twice ( $2 \mathrm{~h}$ per time), then the extract was concentrated under vacuum to $200 \mathrm{~g}$ and stored at $4{ }^{\circ} \mathrm{C}$ for feeding. The group raw dose of E. prostrata (EP) for treating P6 mice was designed as $4.8 \mathrm{~g} \cdot \mathrm{kg}^{-1}$, correspondingly the extract dose was $0.48 \mathrm{~g} \cdot \mathrm{kg}^{-1}$ (Table 2). The above extracts were dissolved into water for regularly feeding per mice per day for 4 weeks $(E P, n=10)$ and another ten mice were chow fed as control group (control, $\mathrm{n}=10$ ). All mice in this study meet the SPF level nutrition needs.

\subsection{Screening and Identification of Endophytes of E. prostrata}

Each $50 \mu \mathrm{L}$ of supernatant were coated on LB and MRS plates and cultured at $37^{\circ} \mathrm{C}$ for $24 \mathrm{~h}$. By observing the colony characteristics on the plates, different phenotypes of bacterial colonies were selected from the plate for identification. The genomic DNA of strains was extracted by kit (Tiangen, China) and used as PCR templates. The $16 \mathrm{~S}$ rDNA sequence was amplified and sequenced using universal primers 27F: 5'-AGAGTTTGATCATGGCTCAG-3' and 1492R: 5'-ACGGTTACCTTGTTACGACTT-3'. The amplification was conducted by polymerase chain reaction in a PCR thermal cycler (Bio-Rad, USA). The PCR amplification system and reaction factor was according to previous work [16]. The amplified products were detected by agarose gel electrophoresis and then purified by kit (Tiangen, China). The $16 \mathrm{~S}$ rDNA sequence of each isolate was then sequenced by BGI Biotech Co., Ltd. (Beijing, China). According to sequencing results, the closest relative was searched in GenBank database using NCBI-nBLAST.

\subsection{Antimicrobial Activity and Survival Ability in Gastrointestinal Tract Tests of Endophytes of $E$. prostrata}

$E$. coli and $S$. aureus were used as the indicator strains for the following assay to detect the antimicrobial activity of the screening strains according to Zhao et al. [16]. The sterilized water and $0.4 \mathrm{mg} / \mathrm{mL}$ gentamicin were used as negative control and positive control, respectively. Plates were cultivated at $37^{\circ} \mathrm{C}$ for $24 \mathrm{~h}$, and antimicrobial circle diameter was measured. Each experiment was repeated 3 times.

Table 2. Dose of E. prostrata for each group.

\begin{tabular}{cccc}
\hline Group & Mice (feeding) & Raw dose & Extract dose \\
\hline control & C57BL/6J (chow-fed) & - & - \\
Pre-EP & C57BL/6J (chow-fed) & - & - \\
Post-EP & C57BL/6J (chow + E. prostrata-fed) & $4.8 \mathrm{~g} \cdot \mathrm{kg}^{-1}$ & $0.48 \mathrm{~g} \cdot \mathrm{kg}^{-1}$ \\
\hline
\end{tabular}


Survival ability in gastrointestinal tract tests included acidic tolerance and bile salt tolerance experiments. MRS medium with $\mathrm{pH}$ values of 3.0, 4.0, 5.0 and 7.0 (control) was prepared with $37 \%$ hydrochloric acid (based on the measured values after autoclave) were used for acidic tolerance test. $0.5 \%, 1 \%$ and $2 \%$ of bile salt was added into MRS medium for bile salt tolerance test, and the medium without bile salt was used as control. Acidic tolerance and bile salt tolerance tests were evaluated by plating and colony counting after $3 \mathrm{~h}$ of cultivation. The survival rate was calculated by the colony number of experimental group divided that of control group.

\subsection{Fecal Sample Collection and Illumina Sequencing}

Fresh stool samples of each group ( 3 of 10 mice per group) were collected in sterile tubes, then frozen and stored at $-80^{\circ} \mathrm{C}$. Afterwards, total genome DNA was extracted by kit (Tiangen, China) from samples and monitored by agarose gels. Afterwards, DNA samples were diluted to $1 \mathrm{ng} \cdot \mu \mathrm{L}^{-1}$ using sterile water and then sent for sequencing (Novogene, Beijing, China). The bacterial 16S rRNA gene V4 region was amplified by PCR with primers using 515F 5'-GTGCCAAGCMG CCGCGGTAA-3' and 806R 5'-GGACTACHVGGGTWTCTAAT-3'. All PCR reactions were carried out and PCR products were conducted according to Zhao et al. [15], the library was sequenced on an Illumina HiSeq 2500 platform and average 250 bp paired-end reads were generated.

\subsection{Bioinformatics Analysis}

Raw tags were performed to QIIME quality controlled process (V1.7.0) under specific filtering conditions to obtain the high-quality clean tags (effective tags) [17]. Operational taxonomic units (OTUs) were clustered with $\geq 97 \%$ sequence similarity by Uparse software (Uparse v7.0.1001, USA) [18]. Representative sequence for each OTU was screened in Green Gene Database for taxonomic annotation [19]. Different taxonomic levels classified from OTUs were conducted using the MUSCLE software (V3.8.31, USA) [20]. Subsequent analysis of alpha diversity (including observed-species and chaol index) and beta diversity (including Principal coordinate analysis (PCoA)) were all performed basing on this output normalized data and further statistically calculated with QIIME and displayed with R software (V2.15.3). The enriched and significant bacteria in each group were identified by linear discriminant analysis (LDA) combined with effect-size measurements (LEfSe), with $\mathrm{P}<0.05$ [15].

\section{Results}

\subsection{Morphology Characteristics of Endophytes in Different Location of Plant E. prostrata}

Five strains of bacteria were screened from the roots, two from the stems and three from the leaves of E. prostrata (Table 1). Among them, the bacterial colonies screened from roots and leaves had common characteristics of white, round, 
smooth and opaque surface, neat edges and Gram staining positive. By morphological analysis, these bacteria are similar to the most common rhizosphere bacteria, Bacillus spp. In addition, there was colony of EP07 screened from stems with round, yellow, edge neat, low convex, smooth and Gram negative characteristics, which has been rarely reported.

\subsection{S Ribosomal DNA Sequence Analysis}

The $16 \mathrm{~S}$ rDNA sequence of the amplified PCR product was determined (Figure 1). Sequences of $1500 \mathrm{bp}$ fragments (supplementary data S1) showed similarity (minimum identity 98\%) with the responding strains blasting in Genbank, and the highest homology strains were showed in Table 1. Both molecular and phenotypic characterization showed that the all the ten strains screened from $E$. prostrata might belong to Bacillus sp., Lactococcus sp., Enterococcus sp., EXiguobacterium sp., and Pantoea sp..

\subsection{Bacteriostatic Activity of Endophytes of E. prostrata}

By oxford cup method, the bacteriostasis activity of 10 strains of bacteria isolated and identified was tested. Strain EP01, 03, 05 showed strong antimicrobial activity against $E$. coli and $S$. aureus (shown in Figure 2). Strain EP02, 04, 06, 07, 08, 09 and 10 have no bacteriostasis to these two pathogenic bacteria. Inhibitory zone of strain with antimicrobial activity isolated from E. prostrata was shown in Table 3. Combining with previous studies, strains with inhibitory activity are widely distributed in roots, stems and leaves. The bacteriostatic activity of strain EP01 is stronger than that of others. However, strain EP08 and EP09 were also Bacillus, which have no inhibitory activity. The antibiotic gentamicin in positive control group was more sensitive to $E$. coli and $S$. aureus.

\subsection{Survival Potential of Endophytes in Gastrointestinal Tract}

Based on the gastrointestinal environment, medium with high concentration of

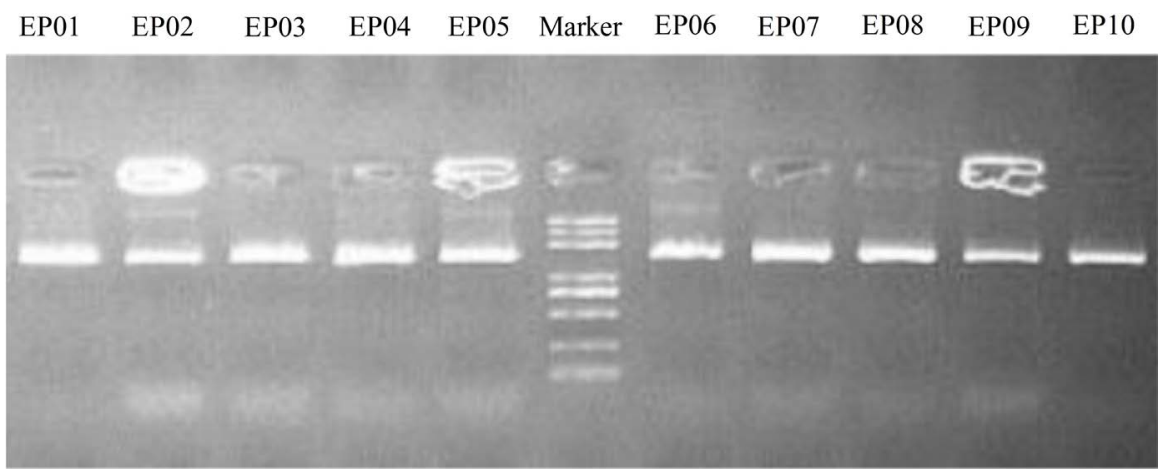

Figure 1. Agarose gel electrophoresis of aimed DNA detected from strain EP01-10. Electrophoresis conditions: 3 ul sample $+1 \%$ agarose gel, Marker band composition: $100 \mathrm{bp}$, $250 \mathrm{bp}, 500 \mathrm{bp}, 750 \mathrm{bp}, 1000 \mathrm{bp}, 2000 \mathrm{bp}, 3000 \mathrm{bp}, 5000 \mathrm{bp}$. The concentration of $750 \mathrm{bp}$ bands was $60 \mathrm{ng} / 3 \mathrm{uL}$, which showed a bright band, and the other bands were $30 \mathrm{ng} / 3 \mathrm{uL}$. The direction of electrophoresis is from top to bottom. 

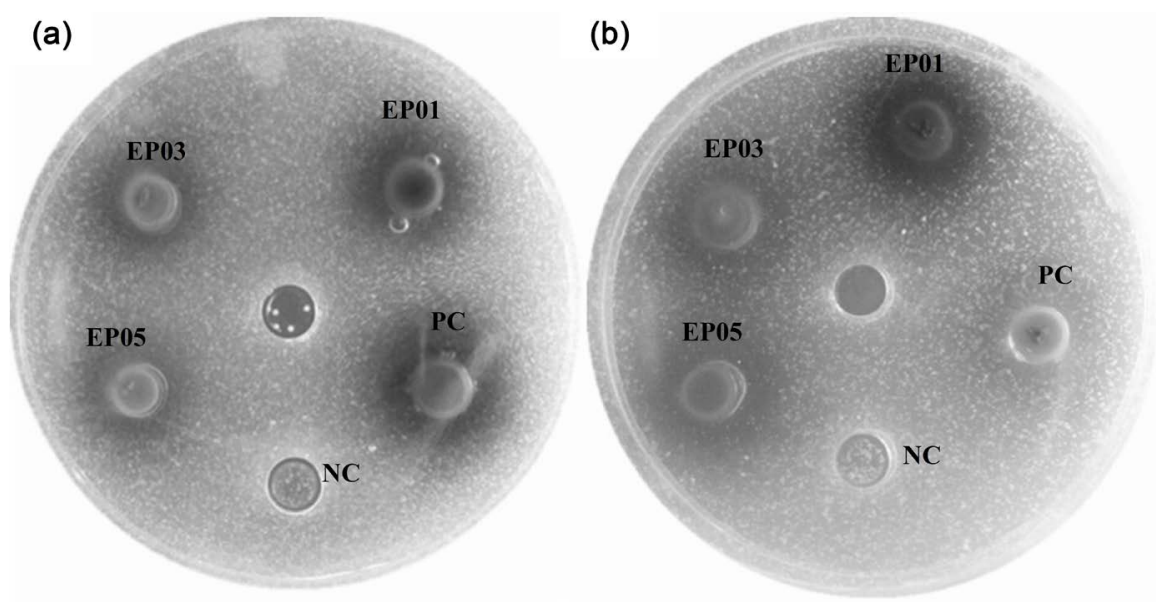

Figure 2. Antimicrobial activity of strains isolated from E. prostrata. Indicator strains were $E$. coli (a) and $S$. aureus (b). NC, negative control miliQ water; PC, $0.4 \mathrm{mg} / \mathrm{mL}$ gentamicin.

Table 3. Inhibitory zone of strains isolated from E. prostrata.

\begin{tabular}{ccccc}
\hline \multirow{2}{*}{ Indicator } & \multicolumn{4}{c}{ Inhibition zone (mm) } \\
\cline { 2 - 5 } & EP01 & EP03 & EP05 & PC \\
\hline S. aureus ATCC6358 & $16.33 \pm 0.21$ & $16.77 \pm 0.36$ & $17.66 \pm 0.12$ & $12.43 \pm 0.04$ \\
E. coli CMCC44103 & $16.32 \pm 0.45$ & $13.51 \pm 0.01$ & $14.38 \pm 0.21$ & $23.21 \pm 0.09$ \\
\hline
\end{tabular}

acid and bile salt were used for survival test. Considering that survival rate of bacteria under the condition of $\mathrm{pH} 7.0$ and without bile salt was 100\% (control), survival rate under other condition was calculated as colony numbers divided by control (Figure 3). Under pH 3.0, the survival rate of strain EP01, 02, 04, 08 and 10 was decreased to $>60 \%$ after $3 \mathrm{~h}$ of cultivation, while the survival rate was $>80 \%$ under $\mathrm{pH} 4$. After growing with $0.5 \%, 1 \%$ and $2 \%$ bile salts for $3 \mathrm{~h}$, the number of EP01, 02, 03, 04, 08, 09 and 10 did not decrease compared with the number without bile salts. All in all, endophytes including EP01, 02, 04, 08 and 10 have the ability to survive in the gastrointestinal tract.

\subsection{Bacterial Community of Gut Microbiota Altered by $E$. prostrata}

By $16 \mathrm{~S}$ rRNA sequencing, an average of 732 OTU clusters were obtained. We found that the GM diversity of Post-EP mice was higher than that of Pre-EP mice by calculating observed species and Chao 1 of alpha diversity index (Figure 4(a)). Unweighted uniFrac-based PCoA of beta diversity revealed a distinct clustering of microbiota composition for each group (Figure 4(b)). Diversity analysis indicated remarkable differences of GM between Pre-EP and Post-EP. At the phylum level, Firmicutes, Bacteroidetes and Proteobacteria constituted the three dominant phyla in all samples (Figure 4(c)). In Post-EP group, the abundance of Firmicutes was significantly $(\mathrm{p}<0.01)$ more than that of Pre-EP (Figure 4(d)). 

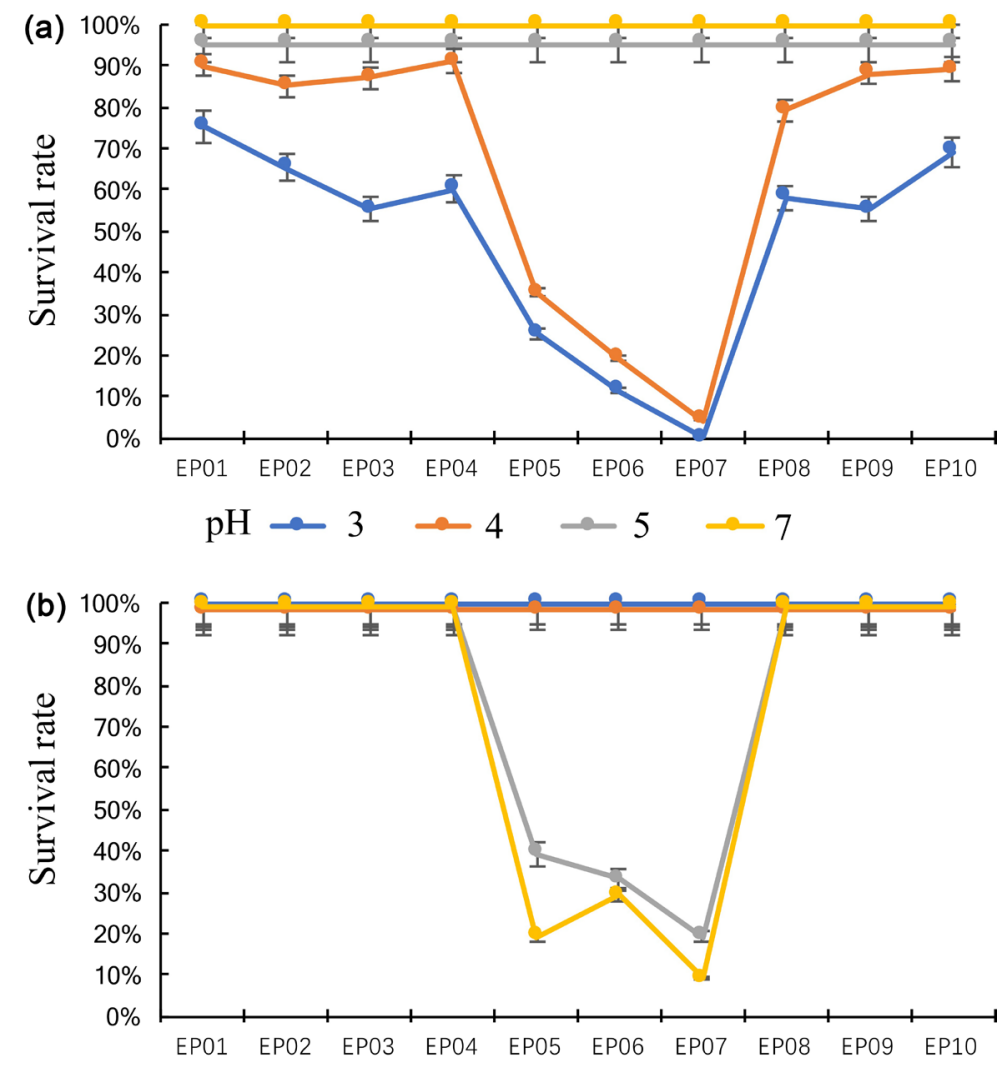

Bile salt $(\%) \rightarrow 0 \multimap 0.5 \multimap 1.0 \multimap 2.0$

Figure 3. (a) Acid tolerance and (b) bile salt tolerance tests of endophytes of E. prostrata.

\subsection{E. prostrata Modulates Gut Microbiota by Targeting Lactococcus Identified as an Endophyte}

Heat map showed top 20 genus levels abundant in this study (Figure 5(a)). In the GM of EP-feeding mice (Post-EP), bacterial genera including Odoribacter, Atopobium, Prevotellaceae UCG-004, Lactococcus and Lactobacillus were more than the proportion of Pre-EP group; and bacterial genera including Prevotellaceae UCG-001, Bacteriodes, Blautia, Clostridium, Allobaculum and Faecalibaculum were less than the proportion of Pre-EP group. We further applied LDA combined LEfSe to explore the biomarkers in each group and relative richness of the bacterial community at various levels. Figure 5(b) summarizes the enrichment and variations in bacterial community in each group. At the genus level, Bacteriodes, Clostridium, Allobaculum and Faecalibaculum were enriched in group before treatment; Lactobacillus, Atopobium, Prevotellaceae UCG-004, Lactococcus and Solobacterium were enriched in the mice group treated with $E$. prostrata. Compared with Pre-EP group, the abundance of genus Lactococcus was higher than that of Post-EP group. Among Lactococcus, the abundance of species including $L$. garvieae, $L$. raffinolactis and other species was remarkably increased after E. prostrata treatment. However, Lactococcus lactis showed the opposite. Thus, as an endophyte, Lactococcus was an important target of the plant in intestinal tract (Figure 5(c)). 


\section{Discussion}

As a TCM, E. prostrata has a significant regulatory effect on GM. Recent study has shown that E. prostrata could improve the mice bone health by altering GM [15]. The bacterial diversity was enriched and the microflora structure were

(a)

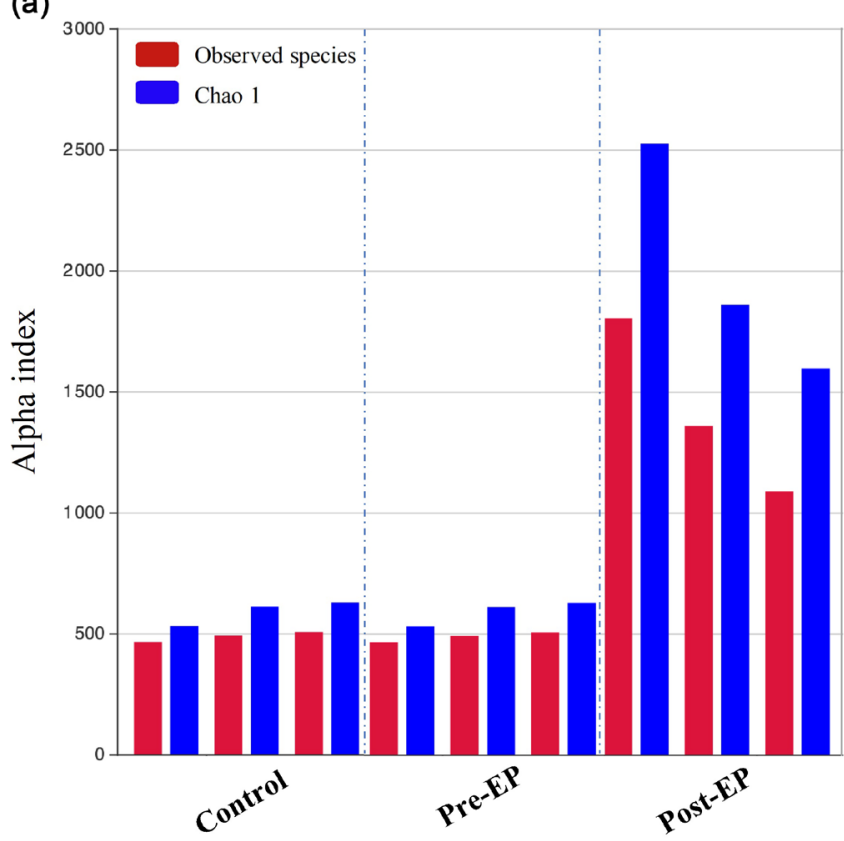

(b)

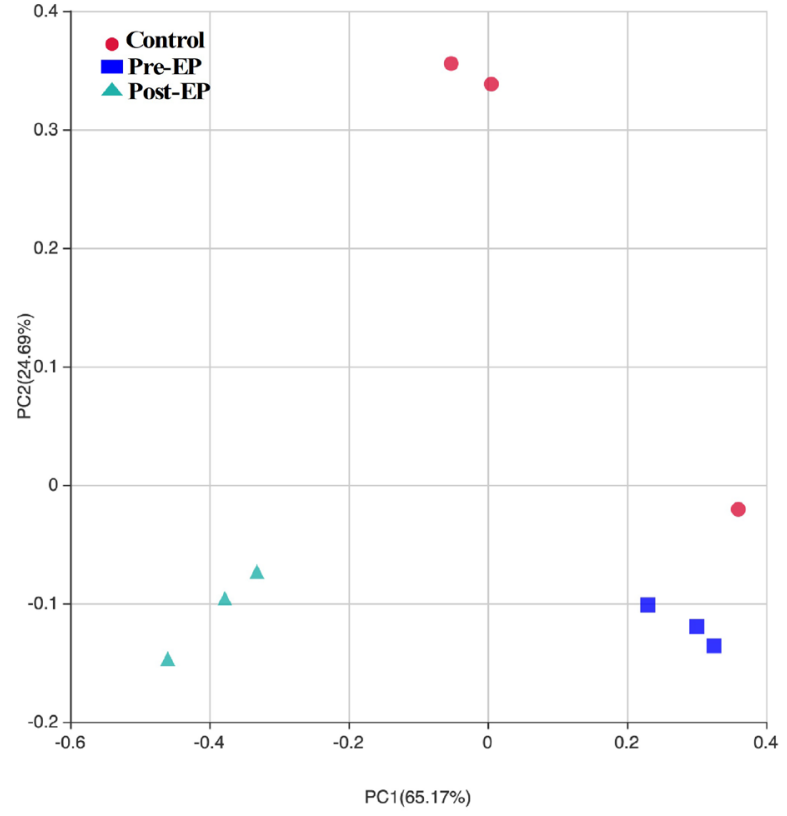

(c)

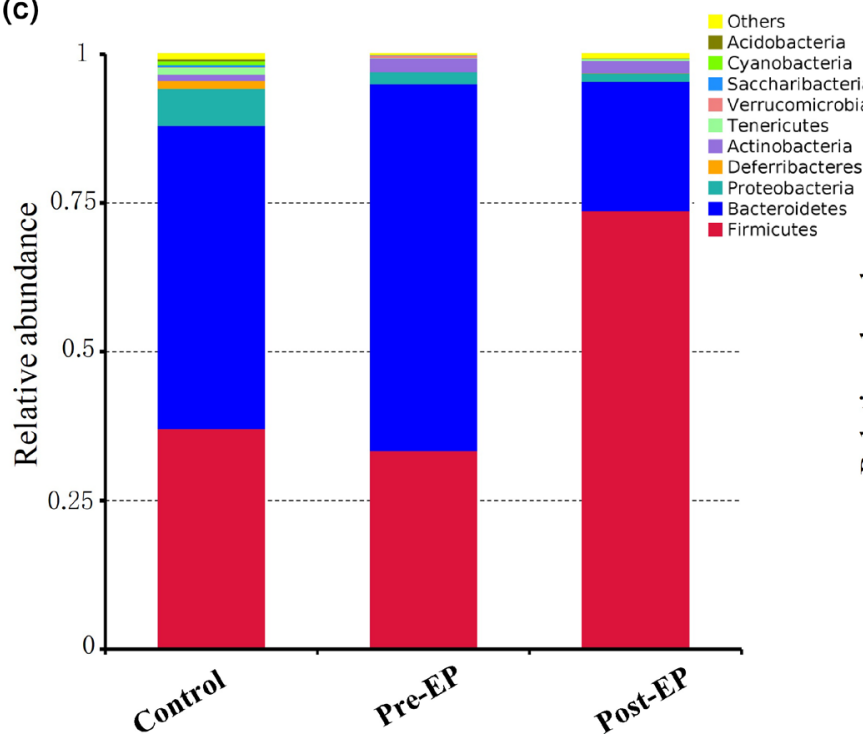

(d)

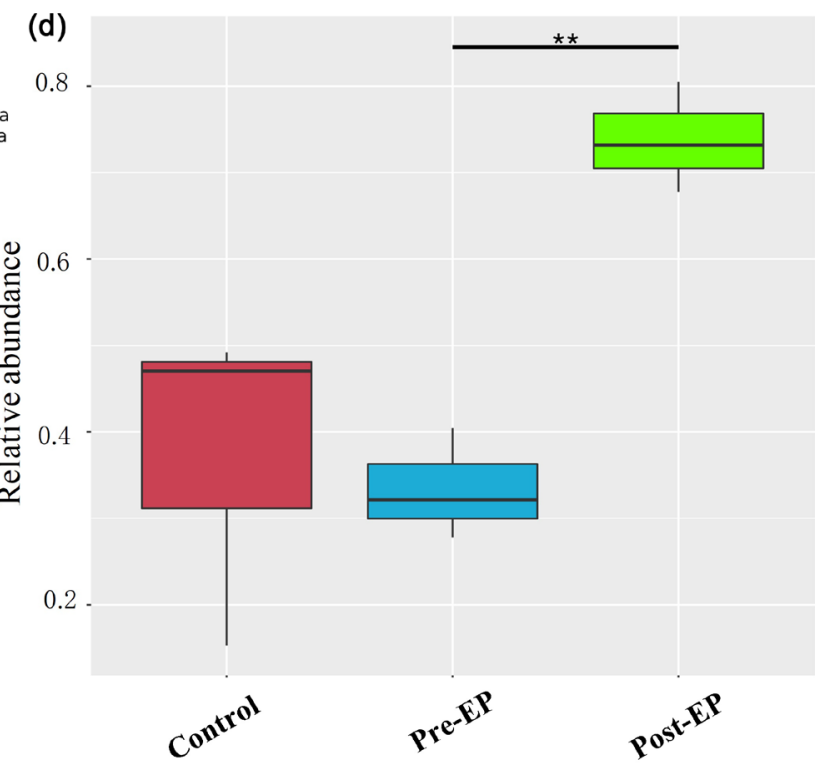

Figure 4. A diversity analysis of microbiota composition for each treatment group. Alpha diversity analysis of (a) Observed species and Chao1; (b) Weighted UniFrac-based principal coordinates analysis (PCoA). The x-axis represents a principal component, and the $y$-axis represents another principal component, and the percentage represents the contribution of the principal component to the sample difference; each point in the fig. represents a sample, and the same group of samples is represented by the same color; (c) The relative bacterial abundance of each group at the phylum level; (d) MetaStat statistical analysis of phylum significance difference between groups. The horizontal axis is the sample grouping; the vertical axis is the relative abundance of corresponding species. "*" means the difference between the two groups is significant $(\mathrm{P}<0.05)$, "“**" means the difference between the two groups is very significant $(\mathrm{P}<0.01)$. 
(a)

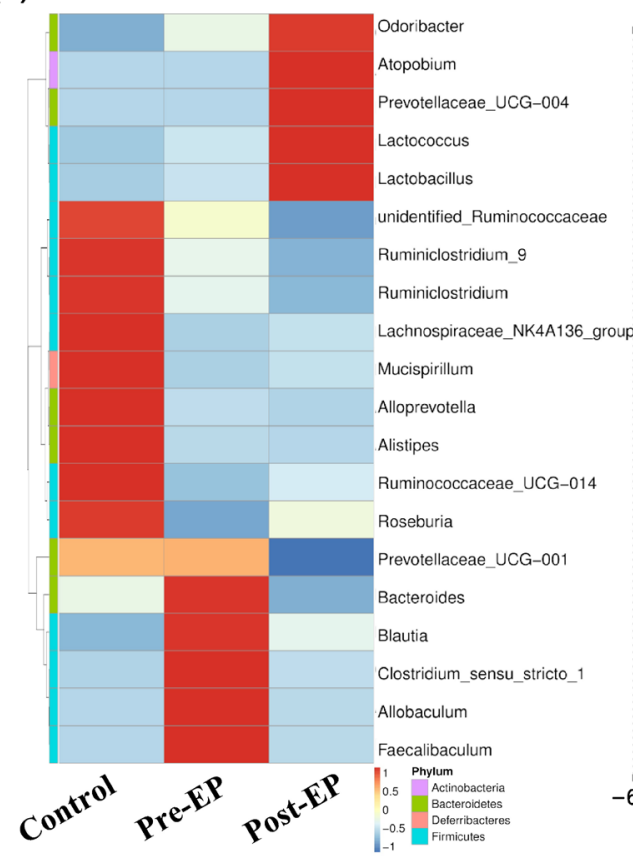

(b)

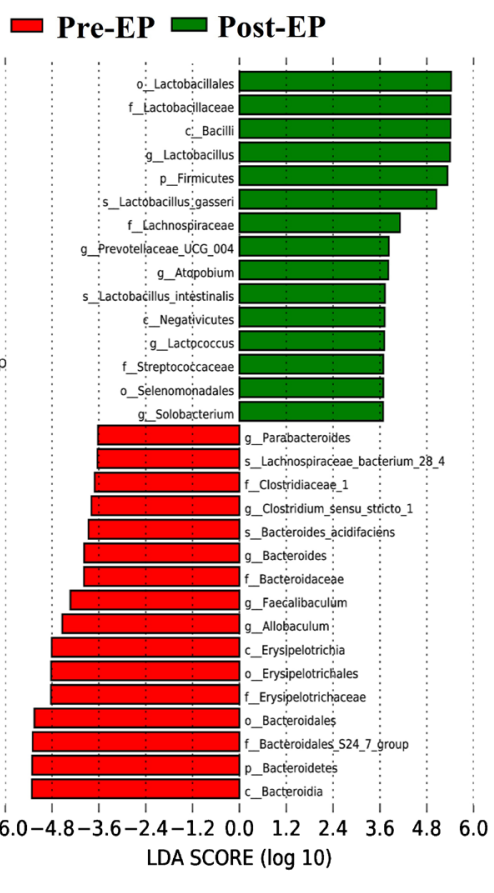

(c)
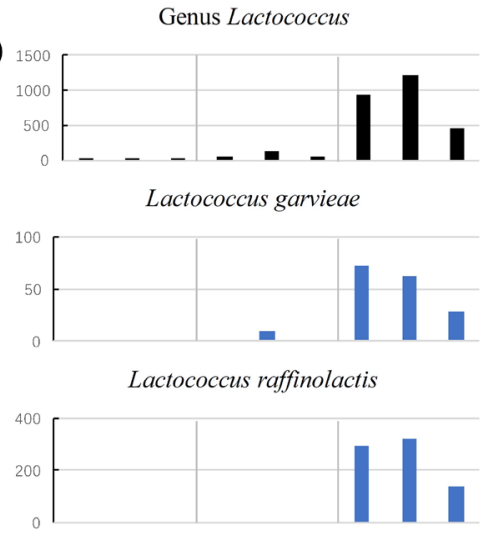

Lactococcus lactis

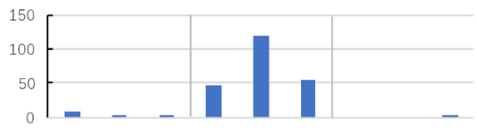

Others Lactococcus species

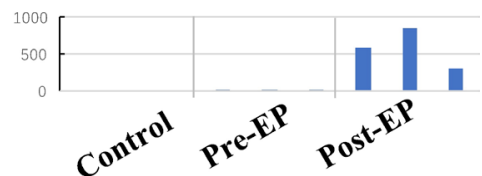

Figure 5. (a) The relative abundance of bacterial communities at genus level; (b) LEfSe analysis result of Post- and Pre-EP group. The species with different abundance are shown. The length of the histogram represents the size of the different species (i.e. LDA score); (c) The absolute abundance of Lactococcus genus and species in each group.

altered after E. prostrata treatment. E. prostrata had the effect of improving the phylum Firmicutes and genus Lactobacillus, Lactococcus and Atopobium of different animal models, indicating that they might be the potential target of $E$. prostrata via GM. Moreover, Lactococcus strain was isolated from plant E. prostrata and had the ability to survive in the gut. However, some endophytes of $E$. prostrata cannot be isolated in lab, or detected by $16 \mathrm{~S}$ sequencing, which limits the study of endophytes of $E$. prostrata efficacy on GM.

Compared with endophytic fungi in TCM, studies on endophytic bacteria in TCM are fewer. Metabolites produced by endophytes, especially antimicrobial substances, can inhibit the growth of pathogens and regulate intestinal disorder [21] [22]. Antimicrobials were reported to contribute to microbiota-induced susceptibility to obesity and metabolic diseases [23]. Gastrointestinal infections are a major concern in human health, but antibiotics cause a harmful effect on GM [24]. Therefore, the anti-infective effect of endophyte-producing antimicrobials is a promising alternative to antibiotics, especially for particular cases where other methods are not allowed (e.g., pregnant women) [25]. Bacillus, Lactococcus and Exiguobacterium isolated in this study are major producers of antimicrobial substances. Lactic acid bacteria (LAB) constitute a group of microorganisms that can produce bacteriocins, which are proteinaceous antimicrobial molecules. According to Alvarez-Sieiro et al [26], putative bacteriocin gene clusters of three lanthipeptides, seven sactipeptides, one LAP, twenty class ii and one class iii were identified in thirteen genomes of Lactococcus strains. In 
addition, two class iii putative bacteriocin gene clusters were identified in four genomes of Exiguobacterium strains. Gene clusters of known and putative bacteriocins, non-ribosomally synthesized peptides (NRPs), polyketides (PKs) and other antimicrobials were also widely distributed in various Bacillus genomes [27].

Probiotics represent one of the fastest growing consumer items on the functional food because they are benefiting human health via GM [28]. Endophytes isolated from edible Chinese herbal medicine can colonized in gut means that it has the potential to develop into probiotics [29]. Therefore, the research and application of endophytic bacteria in traditional Chinese medical plants have broad prospects. Moreover, the interaction of endophytic bacteria and Chinese herbal medicines remains to be studied.

In recent years, the ecological environment has been severely damaged, the predatory utilization of medicinal plants and the difficulty of introduction and cultivation of many wild precious medicinal plants have resulted in serious shortage of natural medicinal resources and lower content of active ingredients. One of the possible reasons is the lack of endophytes, which contribute to form such abundant secondary metabolites for human needs [30]. Although research on endophytes has already attracted people's attention for 20 years, the application of endophytic bacteria in TCM has not drawn too much attention [31] [32]. The combination of the research of endophyte and TCM, especially in resolving resource problems, and has great economic and theoretical value.

\section{Conclusion}

In conclusion, E. prostrata plant is rich in endophytes mainly including Lactococcus and Bacillus, which showed strong antimicrobial activity against E. coli and $S$. aureus, and have the ability to survive in the gastrointestinal tract. $E$. prostrata extract ameliorated the microflora diversity, particularly in increasing the abundance of Lactococcus significantly, indicating that endophyte Lactococcus contributed to E. prostrata modulating GM.

\section{Funding Information}

This work was supported by Program for Tianjin Municipal Education Commission research project (2017KJ142).

\section{Acknowledgements}

We are grateful for the chemical and technical contributions of Dr. Lifeng Han and Professor Lijuan Zhang and would like to thank Mr. Zheng Hu for sequencing help during this study. Xin Zhao was supported by the special fund for "Double First Class" construction of Tianjin University of Traditional Chinese Medicine.

\section{Ethical Statement}

This study was carried out in strict accordance with the recommendations in the 
Guidance Suggestions for the Care and Use of Laboratory Animals issued by the Ministry of Science and Technology of China. The protocols were approved by the Laboratory Animal Ethics Committee of Tianjin University of Traditional Chinese Medicine (Permit Number: TCM-LAEC20180028).

\section{Conflicts of Interest}

The authors declare no conflicts of interest regarding the publication of this paper.

\section{References}

[1] Chang, C.J., Lin, C.S., Lu, C.C., Martel, J., Ko, Y.F., Ojcius, D.M., Tseng, S.F., Wu, T.R., Chen, Y.M., Young, J.D. and Lai, H.C. (2015) Ganoderma lucidum Reduces Obesity in Mice by Modulating the Composition of the Gut Microbiota. Nature Communications, 6, 7489. https://doi.org/10.1038/ncomms8489

[2] Xu, J., Chen, H.B. and Li, S.L. (2017) Understanding the Molecular Mechanisms of the Interplay between Herbal Medicines and Gut Microbiota. Medicinal Research Reviews, 37, 1140-1185. https://doi.org/10.1002/med.21431

[3] El-Deeb, B., Fayez, K. and Gherbawy, Y. (2013) Isolation and Characterization of Endophytic Bacteria from Plectranthus Tenuiflorus Medicinal Plant in Saudi Arabia Desert and Their Antimicrobial Activities. Journal of Plant Interactions, 8, 56-64, https://doi.org/10.1080/17429145.2012.680077

[4] Zhang, L., Chen, Y., Wang, X., Xie, X., Li, Y. and Jin, Z. (2018) The Growth-Promoting Function of Endophytic Bacteria from Panax Notoginseng. Journal of Dalian Polytechnic University, 4.

[5] Brader, G., Compant, S., Mitter, B., Trognitz, F. and Sessitsch, A. (2014) Metabolic Potential of Endophytic Bacteria. Current Opinion in Biotechnology, 27, 30-37. https://doi.org/10.1016/j.copbio.2013.09.012

[6] Huang, J.Y., Zhang, C.J., Yao, Y.L., et al. (2017) Progress in Antimicrobial Substances of Endophytes. Chinese Journal of Biotechnology, 33, 178-186.

[7] Kusari, S. and Spiteller, M. (2011) Are We Ready for Industrial Production of Bioactive Plant Secondary Metabolites Utilizing Endophytes? Natural Product Reports, 28, 1203-1207. https://doi.org/10.1039/c1np00030f

[8] Zhao, L.X., Xu, L.H. and Jiang, C.L. (2012) Methods for the Study of Endophytic Microorganisms from Traditional Chinese Medicine Plants. Methods in Enzymology, 517, 3-21. https://doi.org/10.1016/B978-0-12-404634-4.00001-2

[9] Marquez-Santacruz, H.A., Hernandez-Leon, R., Orozco-Mosqueda, M.C., Velazquez-Sepulveda, I. and Santoyo, G. (2010) Diversity of Bacterial Endophytes in Roots of Mexican Husk Tomato Plants (Physalis ixocarpa) and Their Detection in the Rhizosphere. Genetics and Molecular Research, 9, 2372-2380. https://doi.org/10.4238/vol9-4gmr921

[10] Burdman, S., Jurkevitch, E. and Okon, Y. (2000) Recent Advances in the Use of Plant Growth PROmoting Rhizobacteria (PGPR) in Agriculture. In: Subba Rao, N.S. and Dommergues, Y.R., Eds., Microbial Interactions in Agriculture and Forestry, Science Publishers Inc, Calicut, India, 229-250.

[11] Zhang, N.D., Han, T., Huang, B.K., Rahman, K., Jiang, Y.P. et al. (2016) Traditional Chinese Medicine Formulas for the Treatment of Osteoporosis: Implication for Antiosteoporotic Drug Discovery. Journal of Ethnopharmacology, 189, 61-80. 
[12] Zhuang, X.Y., Yang, J., Li, H.K., Yu, D.M. and Wang, J. (2010) Study on the Mice Model Production of Gastric Hemorrhage Caused by Heat and the Hemostatic Mechanism of Herba Ecliptae. Journal of Mathematical Medicine, 23, 31-33.

[13] Arya, R.K., Singh, A., Yadav, N.K., Cheruvu, S.H., Hossain, Z., et al. (2015) Anti-Breast Tumor Activity of Eclipta Extract In-Vitro and In-Vivo: Novel Evidence of Endoplasmic Reticulum Specific Localization of Hsp60 during Apoptosis. Scientific Reports, 5, 18457.

[14] Han, L., Liu, E., Kojo, A., Zhao, J., Li, W., et al. (2015) Qualitative and Quantitative Analysis of Eclipta prostrata L. by LC/MS. The Scientific World Journal, 2015.

[15] Zhao, X., Ai, J., Mao, H. and Gao, X. (2019) Effects of Eclipta prostrata on Gut Microbiota of SAMP6 Mice with Osteoporosis. Journal of Medical Microbiology, 68, 402-416.

[16] Zhao, X., Zhou, Z.J., Han, Y., Wang, Z.Z., Fan, J. and Xiao, H.Z. (2013) Isolation and Identification of Antifungal Peptides from Bacillus BH072, a Novel Bacterium Isolated from Honey. Microbiological Research, 168, 598-606.

[17] Bokulich, N.A., Subramanian, S., Faith, J.J., Gevers, D., Gordon, J.I. et al. (2013) Quality-Filtering Vastly Improves Diversity Estimates from Illumina Amplicon Sequencing. Nature Methods, 10, 57-59.

[18] Edgar, R.C. (2013) UPARSE: Highly Accurate OTU Sequences from Microbial Amplicon Reads. Nature Methods, 10, 996-998.

[19] DeSantis, T.Z., Hugenholtz, P., Larsen, N., Rojas, M., Brodie, E.L. et al. (2006) Greengenes, a Chimera-Checked $16 \mathrm{~S}$ rRNA Gene Database and Workbench Compatible with ARB. Applied and Environmental Microbiology, 72, 5069-5072.

[20] Edgar, R.C. (2004) MUSCLE: Multiple Sequence Alignment with High Accuracy and High Throughput. Nucleic Acids Research, 32, 1792-1797.

[21] Bäumler, A.J. and Sperandio, V. (2016) Interactions between the Microbiota and Pathogenic Bacteria in the Gut. Nature, 535, 85-93.

[22] Yu, Y.X., Wang, G., Zhao, J.X., Zhang, H. and Chen, W. (2018) Effects of Bacteriocin Produced by Lactic Acid Bacteria on Composition of Gut Microbiota and Metabolites of Gut Flora in Mice. Food and Fermentation Industries, 44, 20-28.

[23] Murphy, E.F., Clarke, S.F., Marques, T.M., Hill, C., Stanton, C., Ross, R.P., O’Doherty, R.M., Shanahan, F. and Cotter, P.D. (2013) Antimicrobials: Strategies for Targeting Obesity and Metabolic Health? Gut Microbes, 4, 48-53.

[24] Ouwehand, A.C., Forssten, S., Hibberd, A.A., Lyra, A. and Stahl, B. (2016) Probiotic Approach to Prevent Antibiotic Resistance. Annals of Medicine, 48, 246-55.

[25] Hammami, R., Fernandez, B., Lacroix, C. and Fliss, I. (2013) Anti-Infective Properties of Bacteriocins: An Update. Cellular and Molecular Life Sciences, 70, 2947-2967.

[26] Alvarez-Sieiro, P., Montalbán-López, M., Mu, D. and Kuipers, O.P. (2016) Bacteriocins of Lactic Acid Bacteria: Extending the Family. Applied Microbiology and Biotechnology, 100, 2939-2951.

[27] Zhao, X. and Kuipers, O.P. (2016) Identification and Classification of Known and Putative Antimicrobial Compounds Produced by a Wide Variety of Bacillales Species. BMC Genomics, 17, 882.

[28] Markowiak, P. and Śliżewska, K. (2017) Effects of Probiotics, Prebiotics, and Synbiotics on Human Health. Nutrients, 9, e1021.

[29] Mastan, A., Rane, D., Dastager, S.G. and Vivek Babu, C.S. (2019) Development of Low-Cost Plant Probiotic Formulations of Functional Endophytes for Sustainable 
Cultivation of Coleus Forskohlii. Microbiological Research, 227, 126310.

[30] Huang, H.J. and Kong, S.Z. (2011) The Research Prospect of Using Traditional Building to Solve RhizomaParidis Recent Heavy Medicinal Resource Shortage. Tropical Forestry, 39, 13-16.

[31] Bhore, S.J., Komathi, V. and Kandasamy, K.I. (2013) Diversity of Endophytic Bacteria in Medicinally Important Nepenthes Species. The Journal of Natural Science, Biology and Medicine, 4, 431-434.

[32] Rhoden, S.A., Garcia, A., Santos e Silva, M.C., Azevedo, J.L. and Pamphile, J.A. (2015) Phylogenetic Analysis of Endophytic Bacterial Isolates from Leaves of the Medicinal Plant Trichilia Elegans A. Juss. (Meliaceae). Genetics and Molecular Research, 14, 1515-1525. 


\section{Supplementary Data S1}

$>$ EP01

GGATTAGCTAGAGCTAGACATGATAGTCGAGCGACGAATCGAGGTACT TGTACCAAGATGAAGAGCGGCGAACGGGTGAGTAACGCGTGGGAAAT CTGCCGAGTAGCGGGGGACAACGTTTGGAAACGAACGCTAATACCGCA TAACAATGGGAATCGCATGATTCTTATTTAAAAGAAGCAAAAGCTTCA CTACTTGATGATCCCGCGTTGTATTAGCTAGTTGGTAGTGTAAAGGAC TACCAAGGCGATGATACATAGCCGGCCTGAGAGGGTGAACGGCCACA CTGGGACTGAGACACGGCCCAGACTCCTACGGGAGGCAGCAGTAGGG AATCTTCGGCAATGGACGAAAGTCTGACCGAGCAACGCCGCGTGAGTG AAGAAGGTTTTCGGATCGTAAAACTCTGTTGTTAGAGAAGAACGTTAA GTAGAGTGGAAAGTTACTTAAGTGACGGTATCTAACCAGAAAGGGACG GCTAACTACGTGCCAGCAGCCGCGGTAATACGTAGGTCCCGAGCGTTG TCCGGATTTATTGGGCGTAAAGCGAGCGCAGGTGGTTTCTTAAGTCTG ATGTAAAAGGCAGTGGCTCAACCATTGTGTGCATTGGAAACTGGGGAA CTTGAGTGCAGGAGAGGGGAGTGGAATTCCATGTGTAGCGGTGAAAT GCGTAGATATATGGAGGAACACCGGAGGCGAAAGCGGCTCTCTGGCCT GTAACTGACACTGAGGCTCGAAAGCGTGGGGAGCAAACAGGATTAGA TACCCTGGTAGTCCACGCCGTAAACGATGAGTGCTAGCTGTAGGGAGC TATAAGTTCTCTGTAGCGCAGCTAACGCATTAAGCACTCCGCCTGGGG AGTACGACCGCAAGGTTGAAACTCAAAGGAATTGACGGGGGCCCGCA CAAGCGGTGGAGCATGTGGTTTAATTCGAAGCAACGCGAAGAACCTTA CCAGGTCTTGACATCCCGATGCAATCCTTAGAGATAAGGAGTTACTTC GGTACATCGGTGACAGGTGGTGCATGGTTGTCGTCAGCTCGTGTCGTG AGATGTTGGGTTAAGTCCCGCAACGAGCGCAACCCTTATTACTAGTTG CCATCATTAAGTTGGGCACTCTAGTGAGACTGCCGGTGATAAACCGGA GGAAGGTGGGGATGACGTCAAATCATCATGCCCCTTATGACCTGGGCT ACACACGTGCTACAATGGGTGGTACAACGAGTCGCCAACCCGCGAGG GTGCGCTAATCTCTTAAAACCATTCTCAGTTCGGATTGCAGGCTGCAA CTCGCCTGCATGAAGTCGGAATCGCTAGTAATCGCGGATCAGCACGCC GCGGTGAATACGTTCCCGGGCCTTGTACACACCGCCCGTCACACCACG GAAGTTGGGAGTACCCAAAGTAGGTTGCCTAACCGCAAGGAGGGAGG TCATCATAGAGTAGAGGGC

$>$ EP02

GCAAGTCGTACGCTTCTTTTTCCACCGGAGCTTGCTCCACCGGAAAAA GAGGAGTGGCGAACGGGTGAGTAACACGTGGGTAACCTGCCCATCAG AAGGGGATAACACTTGGAAACAGGTGCTAATACCGTATAACAATCGAA ACCGCATGGTTTTGATTTGAAAGGCGCTTTCGGGTGTCGCTGATGGAT GGACCCGCGGTGCATTAGCTAGTTGGTGAGGTAACGGCTCACCAAGGC CACGATGCATAGCCGACCTGAGAGGGTGATCGGCCACATTGGGACTGA GACACGGCCCAAACTCCTACGGGAGGCAGCAGTAGGGAATCTTCGGC AATGGACGAAAGTCTGACCGAGCAACGCCGCGTGAGTGAAGAAGGTT 
TTCGGATCGTAAAACTCTGTTGTTAGAGAAGAACAAGGATGAGAGTAA CTGTTCATCCCTTGACGGTATCTAACCAGAAAGCCACGGCTAACTACG TGCCAGCAGCCGCGGTAATACGTAGGTGGCAAGCGTTGTCCGGATTTA TTGGGCGTAAAGCGAGCGCAGGCGGTTTCTTAAGTCTGATGTGAAAGC CCCCGGCTCAACCGGGGAGGGTCATTGGAAACTGGGAGACTTGAGTGC AGAAGAGGAGAGTGGAATTCCATGTGTAGCGGTGAAATGCGTAGATAT ATGGAGGAACACCAGTGGCGAAGGCGGCTCTCTGGTCTGTAACTGACG CTGAGGCTCGAAAGCGTGGGGAGCAAACAGGATTAGATACCCTGGTA GTCCACGCCGTAAACGATGAGTGCTAAGTGTTGGAGGGTTTCCGCCCT TCAGTGCTGCAGCTAACGCATTAAGCACTCCGCCTGGGGAGTACGACC GCAAGGTTGAAACTCAAAGGAATTGACGGGGGCCCGCACAAGCGGTG GAGCATGTGGTTTAATTCGAAGCAACGCGAAGAACCTTACCAGGTCTT GACATCCTTTGACCACTCTAGAGATAGAGCTTCCCCTTCGGGGGCAAA GTGACAGGTGGTGCATGGTTGTCGTCAGCTCGTGTCGTGAGATGTTGG GTTAAGTCCCGCAACGAGCGCAACCCTTATTGTTAGTTGCCATCATTCA GTTGGGCACTCTAGCAAGACTGCCGGTGACAAACCGGAGGAAGGTGG GGATGACGTCAAATCATCATGCCCCTTATGACCTGGGCTACACACGTG CTACAATGGGAAGTACAACGAGTTGCGAAGTCGCGAGGCTAAGCTAAT CTCTTAAAGCTTCTCTCAGTTCGGATTGCAGGCTGCAACTCGCCTGCAT GAAGCCGGAATCGCTAGTAATCGCGGATCAGCACGCCGCGGTGAATAC GTTCCCGGGCCTTGTACACACCGCCCGTCACACCACGAGAGTTTGTAA CACCCGAAGTCGGTGAGGTAACCTTTTGGAGCCAGCCGCCTAAGGTGG GATAGATGATTGGGGGAAGTCGAACA

$>$ EP03

GGCATGCGCGTGCTATACATGCAGTCGAGCGAACTGATTAGAAGCTTG CTTCTATGACGTTAGCGGCGGACGGGTGAGTAACACGTGGGCAACCTG CCTGTAAGACTGGGATAACTTCGGGAAACCGAAGCTAATACCGGATAG GATCTTCTCCTTCATGGGAGATGATTGAAAGATGGTTTCGGCTATCACT TACAGATGGGCCCGCGGTGCATTAGCTAGTTGGTGAGGTAACGGCTCA CCAAGGCAACGATGCATAGCCGACCTGAGAGGGTGATCGGCCACACT GGGACTGAGACACGGCCCAGACTCCTACGGGAGGCAGCAGTAGGGAA TCTTCCGCAATGGACGAAAGTCTGACGGAGCAACGCCGCGTGAGTGAT GAAGGCTTTCGGGTCGTAAAACTCTGTTGTTAGGGAAGAACAAGTACG AGAGTAACTGCTCGTACCTTGACGGTACCTAACCAGAAAGCCACGGCT AACTACGTGCCAGCAGCCGCGGTAATACGTAGGTGGCAAGCGTTATCC GGAATTATTGGGCGTAAAGCGCGCGCAGGCGGTTTCTTAAGTCTGATG TGAAAGCCCACGGCTCAACCGTGGAGGGTCATTGGAAACTGGGGAACT TGAGTGCAGAAGAGAAAAGCGGAATTCCACGTGTAGCGGTGAAATGC GTAGAGATGTGGAGGAACACCAGTGGCGAAGGCGGCTTTTTGGTCTGT AACTGACGCTGAGGCGCGAAAGCGTGGGGAGCAAACAGGATTAGATA CCCTGGTAGTCCACGCCGTAAACGATGAGTGCTAAGTGTTAGAGGGTT TCCGCCCTTTAGTGCTGCAGCTAACGCATTAAGCACTCCGCCTGGGGA 
GTACGGTCGCAAGACTGAAACTCAAAGGAATTGACGGGGGCCCGCAC AAGCGGTGGAGCATGTGGTTTAATTCGAAGCAACGCGAAGAACCTTAC CAGGTCTTGACATCCTCTGACAACTCTAGAGATAGAGCGTTCCCCTTC GGGGGACAGAGTGACAGGTGGTGCATGGTTGTCGTCAGCTCGTGTCGT GAGATGTTGGGTTAAGTCCCGCAACGAGCGCAACCCTTGATCTTAGTT GCCAGCATTTAGTTGGGCACTCTAAGGTGACTGCCGGTGACAAACCGG AGGAAGGTGGGGATGACGTCAAATCATCATGCCCCTTATGACCTGGGC TACACACGTGCTACAATGGATGGTACAAAGGGCTGCAAGACCGCGAG GTCAAGCCAATCCCATAAAACCATTCTCAGTTCGGATTGTAGGCTGCA ACTCGCCTACATGAAGCTGGAATCGCTAGTAATCGCGGATCAGCATGC CGCGGTGAATACGTTCCCGGGCCTTGTACACACCGCCCGTCACACCAC GAGAGTTTGTAACACCCGAAGTCGGTGGAGTAACCGTAAGGAGCTAGC CGCCTAAGGTGACA

\section{$>$ EP04}

TGGGGGTGCTATACATGCAGTCGAGCGAACTGATTAGAAGCTTGCTTC TATGACGTTAGCGGCGGACGGGTGAGTAACACGTGGGCAACCTGCCTG TAAGACTGGGATAACTTCGGGAAACCGAAGCTAATACCGGATAGGATC TTCTCCTTCATGGGAGATGATTGAAAGATGGTTTCGGCTATCACTTACA GATGGGCCCGCGGTGCATTAGCTAGTTGGTGAGGTAACGGCTCACCAA GGCAACGATGCATAGCCGACCTGAGAGGGTGATCGGCCACACTGGGA CTGAGACACGGCCCAGACTCCTACGGGAGGCAGCAGTAGGGAATCTTC CGCAATGGACGAAAGTCTGACGGAGCAACGCCGCGTGAGTGATGAAG GCTTTCGGGTCGTAAAACTCTGTTGTTAGGGAAGAACAAGTACGAGAG TAACTGCTCGTACCTTGACGGTACCTAACCAGAAAGCCACGGCTAACT ACGTGCCAGCAGCCGCGGTAATACGTAGGTGGCAAGCGTTATCCGGAA TTATTGGGCGTAAAGCGCGCGCAGGCGGTTTCTTAAGTCTGATGTGAA AGCCCACGGCTCAACCGTGGAGGGTCATTGGAAACTGGGGAACTTGAG TGCAGAAGAGAAAAGCGGAATTCCACGTGTAGCGGTGAAATGCGTAG AGATGTGGAGGAACACCAGTGGCGAAGGCGGCTTTTTGGTCTGTAACT GACGCTGAGGCGCGAAAGCGTGGGGAGCAAACAGGATTAGATACCCT GGTAGTCCACGCCGTAAACGATGAGTGCTAAGTGTTAGAGGGTTTCCG CCCTTTAGTGCTGCAGCTAACGCATTAAGCACTCCGCCTGGGGAGTAC GGTCGCAAGACTGAAACTCAAAGGAATTGACGGGGGCCCGCACAAGC GGTGGAGCATGTGGTTTAATTCGAAGCAACGCGAAGAACCTTACCAGG TCTTGACATCCTCTGACAACTCTAGAGATAGAGCGTTCCCCTTCGGGG GACAGAGTGACAGGTGGTGCATGGTTGTCGTCAGCTCGTGTCGTGAGA TGTTGGGTTAAGTCCCGCAACGAGCGCAACCCTTGATCTTAGTTGCCA GCATTTAGTTGGGCACTCTAAGGTGACTGCCGGTGACAAACCGGAGGA AGGTGGGGATGACGTCAAATCATCATGCCCCTTATGACCTGGGCTACA CACGTGCTACAATGGATGGTACAAAGGGCTGCAAGACCGCGAGGTCA AGCCAATCCCATAAAACCATTCTCAGTTCGGATTGTAGGCTGCAACTC GCCTACATGAAGCTGGAATCGCTAGTAATCGCGGATCAGCATGCCGCG 
GTGAATACGTTCCCGGGCCTTGTACACACCGCCCGTCACACCACGAGA GTTTGTAACACCCGAAGTCGGTGGAGTAACCGTAAGGAGCTAGCCGCC TAAGTGACAGA

$>$ EP05

GGTGCTATACATGCAGTCGAGCGCAGGAAACTGACGGAACTCTTCGGA GGGAAGGCAGTGGAATGAGCGGCGGACGGGTGAGTAACACGTAAGGA ACCTGCCTCAAGGATTGGGATAACTCCGAGAAATCGGAGCTAATACCG GATAGTTCAACGGACCGCATGGTCCGCTGATGAAAGGCGCTTCGGCGT CACCTTGAGATGGCCTTGCGGTGCATTAGCTAGTTGGTGGGGTAACGG CCCACCAAGGCGACGATGCATAGCCGACCTGAGAGGGTGATCGGCCA CACTGGGACTGAGACACGGCCCAGACTCCTACGGGAGGCAGCAGTAG GGAATCTTCCACAATGGACGAAAGTCTGATGGAGCAACGCCGCGTGAG TGATGAAGGTTTTCGGATCGTAAAACTCTGTTGTAAGGGAAGAACACG TACGAGAGGAAATGCTCGTACCTTGACGGTACCTTACGAGAAAGCCAC GGCTAACTACGTGCCAGCAGCCGCGGTAATACGTAGGTGGCAAGCGTT GTCCGGAATTATTGGGCGTAAAGCGCGCGCAGGCGGCCTTTTAAGTCT GATGTGAAAGCCCCCGGCTCAACCGGGGAGGGCCATTGGAAACTGGA AGGCTTGAGTACAGAAGAGAAGAGTGGAATTCCACGTGTAGCGGTGA AATGCGTAGAGATGTGGAGGAACACCAGTGGCGAAGGCGACTCTTTG GTCTGTAACTGACGCTGAGGCGCGAAAGCGTGGGGAGCAAACAGGAT TAGATACCCTGGTAGTCCACGCCGTAAACGATGAGTGCTAGGTGTTGG GGGGTTTCCGCCCCTCAGTGCTGAAGCTAACGCATTAAGCACTCCGCC TGGGGAGTACGGCCGCAAGGCTGAAACTCAAAGGAATTGACGGGGAC CCGCACAAGCGGTGGAGCATGTGGTTTAATTCGAAGCAACGCGAAGAA CCTTACCAACTCTTGACATCCCATTGACCGCTTGAGAGATCAAGTTTTC CCTTCGGGGACAATGGTGACAGGTGGTGCATGGTTGTCGTCAGCTCGT GTCGTGAGATGTTGGGTTAAGTCCCGCAACGAGCGCAACCCCTATCCT TAGTTGCCAGCATTCAGTTGGGCACTCTAGGGAGACTGCCGGTGACAA ACCGGAGGAAGGTGGGGATGACGTCAAATCATCATGCCCCTTATGAGT TGGGCTACACACGTGCTACAATGGACGGTACAAAGGGCAGCGAGACC GCGAGGTGGAGCCAATCCCATAAAGCCGTTCCCAGTTCGGATTGCAGG CTGCAACTCGCCTGCATGAAGTCGGAATCGCTAGTAATCGCAGGTCAG CATACTGCGGTGAATACGTTCCCGGGTCTTGTACACACCGCCCGTCAC ACCACGAGAGTTTGCAACACCCGAAGCCGGTGAGGTAACCGCAAGGA GCCAGCCGTCGAAGTGGGAGATTT

$>$ EP06

GAGCTATACATGCAAGTCGAGCGGACAGAAGGGAGCTTGCTCCCGGAT GTTAGCGGCGGACGGGTGAGTAACACGTGGGTAACCTGCCTGTAAGAC TGGGATAACTCCGGGAAACCGGAGCTAATACCGGATAGTTCCTTGAAC CGCATGGTTCAAGGATGAAAGACGGTTTCGGCTGTCACTTACAGATGG ACCCGCGGCGCATTAGCTAGTTGGTGAGGTAACGGCTCACCAAGGCGA 
CGATGCGTAGCCGACCTGAGAGGGTGATCGGCCACACTGGGACTGAG ACACGGCCCAGACTCCTACGGGAGGCAGCAGTAGGGAATCTTCCGCAA TGGACGAAAGTCTGACGGAGCAACGCCGCGTGAGTGATGAAGGTTTTC GGATCGTAAAGCTCTGTTGTTAGGGAAGAACAAGTGCAAGAGTAACTG CTTGCACCTTGACGGTACCTAACCAGAAAGCCACGGCTAACTACGTGC CAGCAGCCGCGGTAATACGTAGGTGGCAAGCGTTGTCCGGAATTATTG GGCGTAAAGGGCTCGCAGGCGGTTTCTTAAGTCTGATGTGAAAGCCCC CGGCTCAACCGGGGAGGGTCATTGGAAACTGGGAAACTTGAGTGCAG AAGAGGAGAGTGGAATTCCACGTGTAGCGGTGAAATGCGTAGAGATG TGGAGGAACACCAGTGGCGAAGGCGACTCTCTGGTCTGTAACTGACGC TGAGGAGCGAAAGCGTGGGGAGCGAACAGGATTAGATACCCTGGTAG TCCACGCCGTAAACGATGAGTGCTAAGTGTTAGGGGGTTTCCGCCCCT TAGTGCTGCAGCTAACGCATTAAGCACTCCGCCTGGGGAGTACGGTCG CAAGACTGAAACTCAAAGGAATTGACGGGGGCCCGCACAAGCGGTGG AGCATGTGGTTTAATTCGAAGCAACGCGAAGAACCTTACCAGGTCTTG ACATCCTCTGACAACCCTAGAGATAGGGCTTTCCCTTCGGGGACAGAG TGACAGGTGGTGCATGGTTGTCGTCAGCTCGTGTCGTGAGATGTTGGG TTAAGTCCCGCAACGAGCGCAACCCTTGATCTTAGTTGCCAGCATTCA GTTGGGCACTCTAAGGTGACTGCCGGTGACAAACCGGAGGAAGGTGG GGATGACGTCAAATCATCATGCCCCTTATGACCTGGGCTACACACGTG CTACAATGGACAGAACAAAGGGCTGCGAGACCGCAAGGTTTAGCCAAT CCCACAAATCTGTTCTCAGTTCGGATCGCAGTCTGCAACTCGACTGCG TGAAGCTGGAATCGCTAGTAATCGCGGATCAGCATGCCGCGGTGAATA CGTTCCCGGGCCTTGTACACACCGCCCGTCACACCACGAGAGTTTGCA ACACCCGAAGTCGGTGAGGTAACCTTTATGGAGCCAGCCGCCGAAGTG $>$ EP07

GTTTGCCCGCTACAGTAGAGATGATCGTGGCTCAGGAAGTCATAACAG CTGTGAACCACGGGTGACGAGTGGCGGACGGGTGAGTAATGTCTGGG GATCTGCCCGATAGAGGGGGATAACCACTGGAAACGGTGGCTAATACC GCATAACGTCGCAAGACCAAAGAGGGGGACCTTCGGGCCTCTCACTAT CGGATGAACCCAGATGGGATTAGCTAGTAGGCGGGGTAATGGCCCACC TAGGCGACGATCCCTAGCTGGTCTGAGAGGATGACCAGCCACACTGGA ACTGAGACACGGTCCAGACTCCTACGGGAGGCAGCAGTGGGGAATATT GCACAATGGGCGCAAGCCTGATGCAGCCATGCCGCGTGTATGAAGAA GGCCTTCGGGTTGTAAAGTACTTTCAGCGGGGAGGAAGGCGGTGCGGT TAATAACCGCGCCGATTGACGTTACCCGCAGAAGAAGCACCGGCTAAC TCCGTGCCAGCAGCCGCGGTAATACGGAGGGTGCAAGCGTTAATCGGA ATTACTGGGCGTAAAGCGCACGCAGGCGGTCTGTTAAGTCAGATGTGA AATCCCCGGGCTTAACCTGGGAACTGCATTTGAAACTGGCAGGCTTGA GTCTTGTAGAGGGGGGTAGAATTCCAGGTGTAGCGGTGAAATGCGTAG AGATCTGGAGGAATACCGGTGGCGAAGGCGGCCCCCTGGACAAAGAC TGACGCTCAGGTGCGAAAGCGTGGGGAGCAAACAGGATTAGATACCC 
TGGTAGTCCACGCCGTAAACGATGTCGACTTGGAGGTTGTTCCCTTGA GGAGTGGCTTCCGGAGCTAACGCGTTAAGTCGACCGCCTGGGGAGTAC GGCCGCAAGGTTAAAACTCAAATGAATTGACGGGGGCCCGCACAAGC GGTGGAGCATGTGGTTTAATTCGATGCAACGCGAAGAACCTTACCTAC TCTTGACATCCACGGAATTCGGCAGAGATGCCTCAGTGCCTTCGGGAA CCGTGAGACAGGTGCTGCATGGCTGTCGTCAGCTCGTGTTGTGAAATG TTGGGTTAAGTCCCGCAACGAGCGCAACCCTTATCCTTTGTTGCCAGC GATTCGGTCGGGAACTCAAAGGAGACTGCCGGTGATAAACCGGAGGA AGGTGGGGATGACGTCAAGTCATCATGGCCCTTACGAGTAGGGCTACA CACGTGCTACAATGGCGCATACAAAGAGAAGCGACCTCGCGAGAGCA AGCGGACCTCACAAAGTGCGTCGTAGTCCGGATCGGAGTCTGCAACTC GACTCCGTGAAGTCGGAATCGCTAGTAATCGTGGATCAGAATGCCACG GTGAATACGTTCCCGGGCCTTGTACACACCGCCCGTCACACCATGGGA GTGGGTTGCAATAAGTAAGTACGGTAGCTTAACCTGTGGGAGCGCAGC TAACCATAGAGGAAATGGC

$>$ EP08

TGCAGTCGAGCGAACTGATTAGAAGCTTGCTTCTATGACGTTAGCGGC GGACGGGTGAGTAACACGTGGGCAACCTGCCTGTAAGACTGGGATAA CTTCGGGAAACCGAAGCTAATACCGGATAGGATCTTCTCCTTCATGGG AGATGATTGAAAGATGGTTTCGGCTATCACTTACAGATGGGCCCGCGG TGCATTAGCTAGTTGGTGAGGTAACGGCTCACCAAGGCAACGATGCAT AGCCGACCTGAGAGGGTGATCGGCCACACTGGGACTGAGACACGGCC CAGACTCCTACGGGAGGCAGCAGTAGGGAATCTTCCGCAATGGACGAA AGTCTGACGGAGCAACGCCGCGTGAGTGATGAAGGCTTTCGGGTCGTA AAACTCTGTTGTTAGGGAAGAACAAGTACGAGAGTAACTGCTCGTACC TTGACGGTACCTAACCAGAAAGCCACGGCTAACTACGTGCCAGCAGCC GCGGTAATACGTAGGTGGCAAGCGTTATCCGGAATTATTGGGCGTAAA GCGCGCGCAGGCGGTTTCTTAAGTCTGATGTGAAAGCCCACGGCTCAA CCGTGGAGGGTCATTGGAAACTGGGGAACTTGAGTGCAGAAGAGAAA AGCGGAATTCCACGTGTAGCGGTGAAATGCGTAGAGATGTGGAGGAA CACCAGTGGCGAAGGCGGCTTTTTGGTCTGTAACTGACGCTGAGGCGC GAAAGCGTGGGGAGCAAACAGGATTAGATACCCTGGTAGTCCACGCC GTAAACGATGAGTGCTAAGTGTTAGAGGGTTTCCGCCCTTTAGTGCTG CAGCTAACGCATTAAGCACTCCGCCTGGGGAGTACGGTCGCAAGACTG AAACTCAAAGGAATTGACGGGGGCCCGCACAAGCGGTGGAGCATGTG GTTTAATTCGAAGCAACGCGAAGAACCTTACCAGGTCTTGACATCCTC TGACAACTCTAGAGATAGAGCGTTCCCCTTCGGGGGACAGAGTGACAG GTGGTGCATGGTTGTCGTCAGCTCGTGTCGTGAGATGTTGGGTTAAGT CCCGCAACGAGCGCAACCCTTGATCTTAGTTGCCAGCATTTAGTTGGG CACTCTAAGGTGACTGCCGGTGACAAACCGGAGGAAGGTGGGGATGA CGTCAAATCATCATGCCCCTTATGACCTGGGCTACACACGTGCTACAA TGGATGGTACAAAGGGCTGCAAGACCGCGAGGTCAAGCCAATCCCATA 
AAACCATTCTCAGTTCGGATTGTAGGCTGCAACTCGCCTACATGAAGC TGGAATCGCTAGTAATCGCGGATCAGCATGCCGCGGTGAATACGTTCC CGGGCCTTGTACACACCGCCCGTCACACCACGAGAGTTTGTAACACCC GAAGTCGGTGGAGTAACCGTAAGGAGCTAGCCGCCTAAG

$>$ EP09

CTATAATGCAAGTCGAGCGAACTGATTAGAAGCTTGCTTCTATGACGT TAGCGGCGGACGGGTGAGTAACACGTGGGCAACCTGCCTGTAAGACT GGGATAACTTCGGGAAACCGAAGCTAATACCGGATAGGATCTTCTCCT TCATGGGAGATGATTGAAAGATGGTTTCGGCTATCACTTACAGATGGG CCCGCGGTGCATTAGCTAGTTGGTGAGGTAACGGCTCACCAAGGCAAC GATGCATAGCCGACCTGAGAGGGTGATCGGCCACACTGGGACTGAGA CACGGCCCAGACTCCTACGGGAGGCAGCAGTAGGGAATCTTCCGCAAT GGACGAAAGTCTGACGGAGCAACGCCGCGTGAGTGATGAAGGCTTTC GGGTCGTAAAACTCTGTTGTTAGGGAAGAACAAGTACGAGAGTAACTG CTCGTACCTTGACGGTACCTAACCAGAAAGCCACGGCTAACTACGTGC CAGCAGCCGCGGTAATACGTAGGTGGCAAGCGTTATCCGGAATTATTG GGCGTAAAGCGCGCGCAGGCGGTTTCTTAAGTCTGATGTGAAAGCCCA CGGCTCAACCGTGGAGGGTCATTGGAAACTGGGGAACTTGAGTGCAGA AGAGAAAAGCGGAATTCCACGTGTAGCGGTGAAATGCGTAGAGATGT GGAGGAACACCAGTGGCGAAGGCGGCTTTTTGGTCTGTAACTGACGCT GAGGCGCGAAAGCGTGGGGAGCAAACAGGATTAGATACCCTGGTAGT CCACGCCGTAAACGATGAGTGCTAAGTGTTAGAGGGTTTCCGCCCTTT AGTGCTGCAGCTAACGCATTAAGCACTCCGCCTGGGGAGTACGGTCGC AAGACTGAAACTCAAAGGAATTGACGGGGGCCCGCACAAGCGGTGGA GCATGTGGTTTAATTCGAAGCAACGCGAAGAACCTTACCAGGTCTTGA CATCCTCTGACAACTCTAGAGATAGAGCGTTCCCCTTCGGGGGACAGA GTGACAGGTGGTGCATGGTTGTCGTCAGCTCGTGTCGTGAGATGTTGG GTTAAGTCCCGCAACGAGCGCAACCCTTGATCTTAGTTGCCAGCATTT AGTTGGGCACTCTAAGGTGACTGCCGGTGACAAACCGGAGGAAGGTG GGGATGACGTCAAATCATCATGCCCCTTATGACCTGGGCTACACACGT GCTACAATGGATGGTACAAAGGGCTGCAAGACCGCGAGGTCAAGCCA ATCCCATAAAACCATTCTCAGTTCGGATTGTAGGCTGCAACTCGCCTA CATGAAGCTGGAATCGCTAGTAATCGCGGATCAGCATGCCGCGGTGAA TACGTTCCCGGGCCTTGTACACACCGCCCGTCACACCACGAGAGTTTG TAACACCCGAAGTCGGTGGAGTAACCGTAAGGAGCTAGCCGCCTAAGG TGACAGA

$>$ EP10

ATGCGCATGCTATAATGCAGTCGAGCGAATGGATTAAGAGCTTGCTCT TATGAAGTTAGCGGCGGACGGGTGAGTAACACGTGGGTAACCTGCCCA TAAGACTGGGATAACTCCGGGAAACCGGGGCTAATACCGGATAACATT TTGAACTGCATGGTTCGAAATTGAAAGGCGGCTTCGGCTGTCACTTAT 
GGATGGACCCGCGTCGCATTAGCTAGTTGGTGAGGTAACGGCTCACCA AGGCAACGATGCGTAGCCGACCTGAGAGGGTGATCGGCCACACTGGG ACTGAGACACGGCCCAGACTCCTACGGGAGGCAGCAGTAGGGAATCTT CCGCAATGGACGAAAGTCTGACGGAGCAACGCCGCGTGAGTGATGAA GGCTTTCGGGTCGTAAAACTCTGTTGTTAGGGAAGAACAAGTGCTAGT TGAATAAGCTGGCACCTTGACGGTACCTAACCAGAAAGCCACGGCTAA CTACGTGCCAGCAGCCGCGGTAATACGTAGGTGGCAAGCGTTATCCGG AATTATTGGGCGTAAAGCGCGCGCAGGTGGTTTCTTAAGTCTGATGTG AAAGCCCACGGCTCAACCGTGGAGGGTCATTGGAAACTGGGAGACTTG AGTGCAGAAGAGGAAAGTGGAATTCCATGTGTAGCGGTGAAATGCGT AGAGATATGGAGGAACACCAGTGGCGAAGGCGACTTTCTGGTCTGTAA CTGACACTGAGGCGCGAAAGCGTGGGGAGCAAACAGGATTAGATACC CTGGTAGTCCACGCCGTAAACGATGAGTGCTAAGTGTTAGAGGGTTTC CGCCCTTTAGTGCTGAAGTTAACGCATTAAGCACTCCGCCTGGGGAGT ACGGCCGCAAGGCTGAAACTCAAAGGAATTGACGGGGGCCCGCACAA GCGGTGGAGCATGTGGTTTAATTCGAAGCAACGCGAAGAACCTTACCA GGTCTTGACATCCTCTGAAAACCCTAGAGATAGGGCTTCTCCTTCGGG AGCAGAGTGACAGGTGGTGCATGGTTGTCGTCAGCTCGTGTCGTGAGA TGTTGGGTTAAGTCCCGCAACGAGCGCAACCCTTGATCTTAGTTGCCA TCATTAAGTTGGGCACTCTAAGGTGACTGCCGGTGACAAACCGGAGGA AGGTGGGGATGACGTCAAATCATCATGCCCCTTATGACCTGGGCTACA CACGTGCTACAATGGACGGTACAAAGAGCTGCAAGACCGCGAGGTGG AGCTAATCTCATAAAACCGTTCTCAGTTCGGATTGTAGGCTGCAACTC GCCTACATGAAGCTGGAATCGCTAGTAATCGCGGATCAGCATGCCGCG GTGAATACGTTCCCGGGCCTTGTACACACCGCCCGTCACACCACGAGA GTTTGTAACACCCGAAGTCGGTGGGGTAACCTTTTGGAGCCAGCCGCC TAAGTGA 